\title{
Reproductive knowledge and use of contraception among women with diabetes
}

\author{
Ayesha Osman, MB ChB, FCOG (SA), MMed (O\&G); Anne Hoffman, RN, RM; Shane Moore, RN, RM; \\ Zephne van der Spuy, MB ChB, PhD, FRCOG, FCOG (SA) \\ Department of Obstetrics and Gynaecology, Faculty of Health Sciences, University of Cape Town and Groote Schuur Hospital, \\ Cape Town, South Africa
}

Corresponding author: A Osman (ayesha2309@gmail.com)

Background. Poorly controlled diabetes is associated with poor maternal and fetal outcomes, yet many women become pregnant before establishing control. Reducing unintended pregnancies is a vital step towards improving perinatal and maternal morbidity and mortality. Objectives. To assess the reproductive knowledge and use of contraception in women of reproductive age attending diabetes outpatient clinics.

Methods. A prospective descriptive study was conducted of women known to have diabetes, aged 18 - 45 years, attending the diabetic clinics at Groote Schuur Hospital or the local community health centres in Cape Town, South Africa. A questionnaire consisting of social, demographic and family details as well as contraceptive use and knowledge was administered.

Results. Some common themes emerged, namely that $44.2 \%$ of the women with previous pregnancies had had unintended pregnancies, and that this was more common among single $(58.8 \%)$ and younger women. Women with type 1 diabetes had better knowledge than those with type 2 diabetes of how pregnancy affects diabetes, but better knowledge did not translate to better contraception use. Despite the fact that 102 participants (88.7\%) attended diabetes clinics two or more times a year, knowledge of pregnancy- and reproductive health-related complications was limited, and only 30 participants (26.1\%) had received advice on contraception at these clinics.

Conclusion. Knowledge about the impact of diabetes on pregnancy and that of pregnancy on diabetes was suboptimal. We recommend that reproductive health services be included at the routine diabetes clinic visit.

S Afr Med J 2015;105(9):760-764. DOI:10.7196/SAMJnew.8170

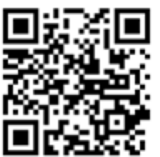

The prevalence of diabetes mellitus is increasing rapidly worldwide. Diabetes currently affects 366 million people globally, and the number is expected to rise to 552 million by the year $2030 .^{[1,2]}$ In South Africa (SA) 3 million men and women have diagnosed diabetes, and there are an estimated further 3 million living with the disease who remain undiagnosed. ${ }^{[1]}$

Fetal complications of maternal diabetes can be divided into two major categories: (i) early complications, reflecting the impact of maternal disease on early fetal development due to poor glucose control during the period of organogenesis in the first trimester; and (ii) late complications, including unexplained stillbirths and macrosomia in the second and third trimesters and hypoglycaemia, respiratory distress syndrome and jaundice in the neonatal period. Direct obstetric complications include spontaneous miscarriage, pre-eclampsia, polyhydramnios and obstructed labour because of macrosomia. ${ }^{[3,4]}$

Maternal complications of pre-existing diabetes can be equally severe, and even life-threatening, but are often ignored by women desperate to have a child.

The 2007 Saving Mothers report stated that $76 \%$ of women with pre-existing maternal disease attended antenatal clinics. ${ }^{[5]}$ The health system is therefore in a good position to intervene and prevent death, yet pre-existing maternal disease $(6 \%)$ remains one of the five leading causes of maternal deaths, both in SA and internationally ${ }^{[5]}$ This suggests that antenatal intervention is too late to initiate optimal care for some patients, and that there is a lack of preconception planning and counselling, which are central to good management.

The 2007 Saving Mothers report stated that the best way to prevent maternal deaths is to prevent pregnancy. ${ }^{[5]}$ One of its recommendations was to promote contraceptive use through education and service provision. This also promotes reproductive health. Women with unplanned pregnancies, especially those with an underlying medical condition such as pregestational diabetes, are at an increased risk of maternal and neonatal complications. Tight glycaemic control for diabetic women in the periconception period is essential to reduce the risks of complications. ${ }^{[6]}$

There are limited SA data on chronic medical diseases and contraceptive use and knowledge, with the exception of HIV. The 2003 South Africa Demographic and Health Survey (SADHS) 2003 data $^{[7]}$ are already more than 10 years old, and we have not been able to find anything more on chronic medical disorders and contraception in recent published research.

\section{Objectives}

To assess the reproductive knowledge of diabetic women of reproductive age attending outpatient clinics for the management of diabetes, and their use of contraception. A secondary objective was to elicit what counselling women with diabetes received on the implications of their condition with regard to pregnancy and reproductive health.

\section{Methods}

\section{Study design and population}

A prospective descriptive study design was used. The study population comprised women aged 18 - 45 years attending diabetes outpatient clinics between 1 March and 31 July 2012.

Recruitment was by convenience sampling, and patients were initially recruited from the three diabetes clinics at Groote Schuur Hospital 
(GSH), Cape Town, SA. The recruitment area was later extended to include community health centres (CHCs) with 'diabetes clubs' (Woodstock, Lady Michaelis, Hanover Park and Gugulethu CHCs).

\section{Data collection}

Data were collected by means of a questionnaire administered by experienced research staff in the Reproductive Medicine Unit, GSH. Additional clinical information was obtained from the medical folder.

The study investigators were not involved in the medical management of the patients. Interviews were conducted in private rooms to maintain strict confidentiality, and personal identifying information was not entered into the database. The questionnaire consisted of demographic and social details of patients as well as medical and obstetric history, knowledge about reproduction and contraception use.

\section{Consent}

Approval for the study was granted by the Human Research Ethics Committee of the Faculty of Health Sciences, University of Cape Town (HREC REF: 500/2011) and the Provincial Health Research Council of the Western Cape (RP 48/2012). All participants provided signed informed consent before the questionnaire was administered.

\section{Data management and statistical analysis}

Data were processed in the Reproductive Medicine Unit. All data were double-entered into a database created using Microsoft Office Excel 2007 and then cross-checked using the statistical analysis programme Stata. Statistical analysis was performed using Stata software version 11 (StataCorp 2009, USA), with assistance from the Department of Statistical Sciences of the University of Cape Town.

Demographic details were presented in a descriptive manner. In the analysis of continuous data such as age and parity, which are non-normally distributed, we used the Mann-Whitney $U$-test/Wilcoxon rank sum test for formal comparisons. In the case of categorical variables, cross-tabulations were used together with $\chi^{2}$ tests of association and Fisher's exact tests where appropriate. The level of significance was set as $p<0.05$.

\section{Results \\ Patient characteristics}

A total of 115 women aged 18 - 45 years were recruited. No patient declined to participate.

Less than half of the participants were employed $(41.7 \%, n=48)$, but no woman identified herself as being without financial support. Of note, 19 participants were receiving disability grants and four received child support grants which they cited as financial support. The majority of the participants lived in a dwelling with access to basic amenities (water, electricity, plumbing), and less than $10 \%(n=11)$ lived in an informal dwelling without these amenities.

Sixty-two respondents (53.9\%) had completed a minimum of grade 12 level of education, and only four (3.5\%) had less than a grade 7 education. Sixty-seven women (58.3\%) were either married or in a stable relationship (Fig. 1). Only one participant was unable to identify a source of emotional support.

\section{Obstetric history}

The 77 women who had previously been pregnant had a total of 180 pregnancies between them. Of these women, 34 (44.2\%) reported some of their pregnancies as unintended. The Mann-Whitney $U$-test/Wilcoxon rank sum test demonstrated an association between age and intended pregnancy, unintended pregnancy tending to be associated with younger age $(z=2.308, p=0.0210)$.

Single women were also more likely to have had unintended pregnancies, with 20 of the reported 34 unintended pregnancies (58.8\%) having been in women who classified themselves as single (Pearson $\chi^{2}(6)=16.8552$, Fisher's exact $p=0.003)$. The remaining 14 women, who were currently married, did not specify whether the unintended pregnancy had occurred before or after marriage.

Only 10 of the 43 women (23.3\%) who reported having had an intended pregnancy identified themselves as single; the remaining $33(76.7 \%)$ were currently married, divorced or separated, but as their marital status at the

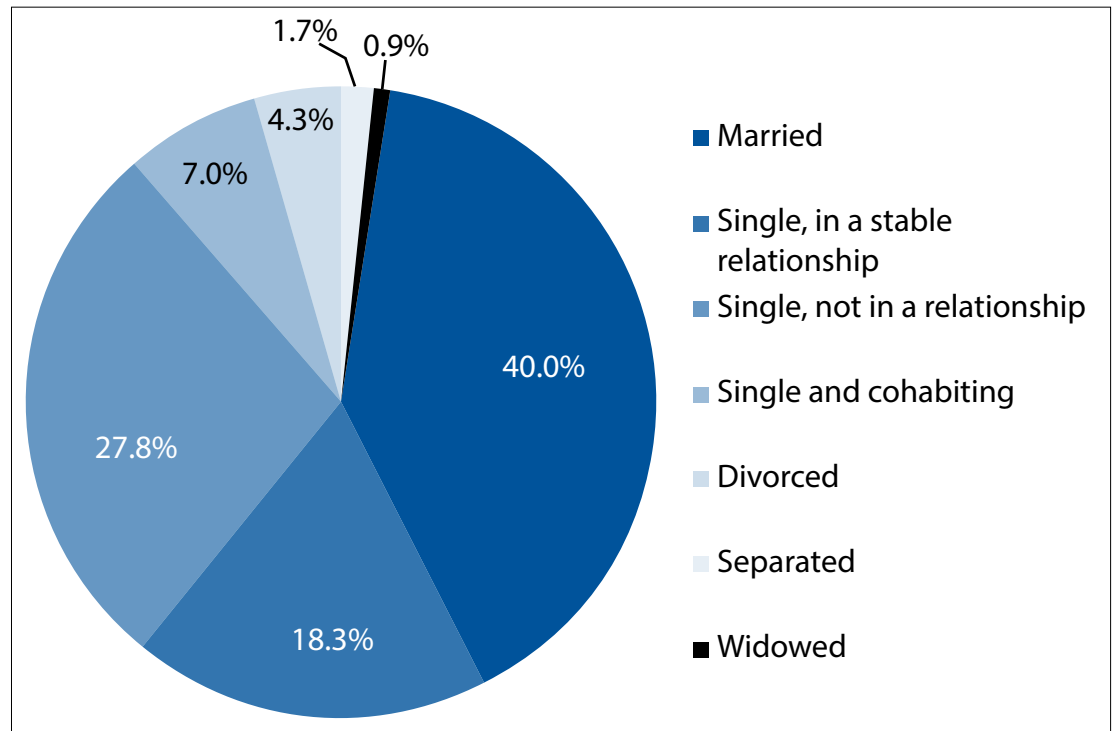

Fig. 1. Marital status of the study subjects.

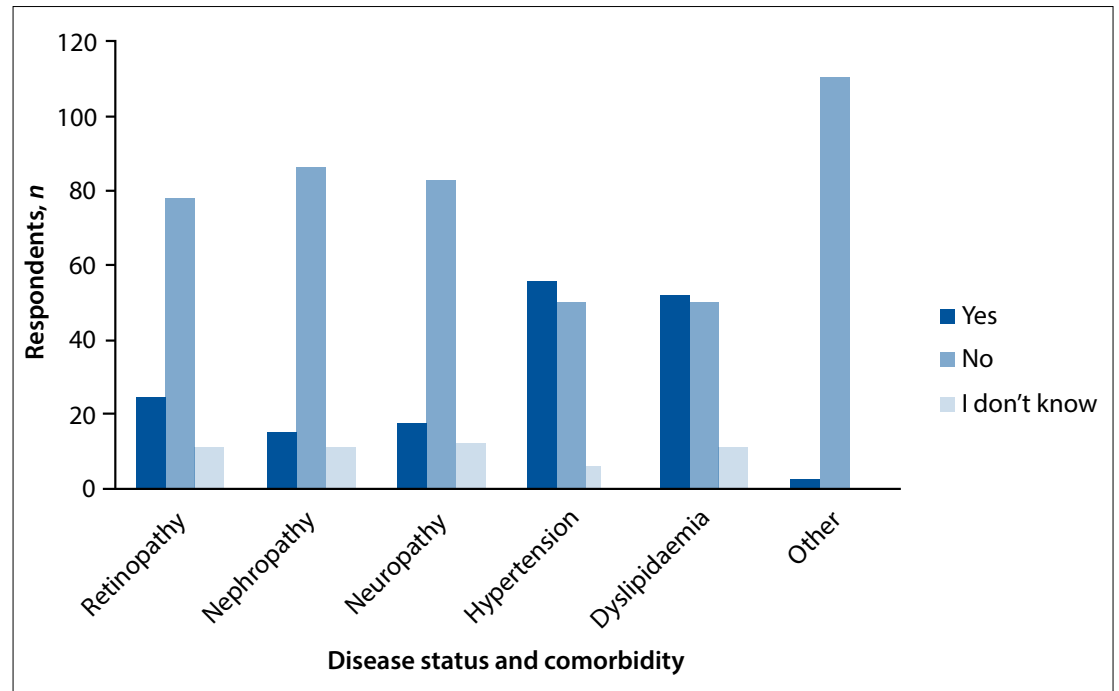

Fig. 2. Current status of disease and comorbidity. (Other = amputations, heart attacks, heart failure, comas, strokes, poor healing.) 
time of the pregnancy was not specified, this information should be interpreted with caution.

We found no association between educational level and emotional support and intended v. unintended pregnancies (Pearson $\chi^{2}(9)=10.6226$, Fisher's exact $p=0.250$ and Pearson $\chi^{2}(1)=0.8011$, Fisher's exact $p=1.000$, respectively.)

A high proportion of the respondents who had been pregnant reported that they had been offered contraception after the birth of the baby (151 of 180 pregnancies, $83.9 \%$ ). Despite this high number, in the group of 34 women with unintended pregnancies, 24 were not using any contraception at the time of conception.

\section{Medical history}

Sixty-one participants $(53.0 \%)$ had type 1 diabetes (requiring insulin from the time of diagnosis), and 70 were using additional medication such as antihypertensives and statins.

Eighty-five women (73.9\%) had comorbid conditions or complications secondary to diabetes (most commonly hypertension and dylipidaemia) (Fig. 2). We found no association between complications of diabetes or comorbid disease and current or previous use of contraception (Pearson $\chi^{2}(1)=0.1780$, Fisher's exact $p=0.826$ ).

Patients with type 1 diabetes did not differ from those with type 2 diabetes with regard to number of intended pregnancies and unintended pregnancies (Pearson $\chi^{2}(1)=1.9057$, Fisher's exact $\left.p=0.248\right)$. We also found no association between type of diabetes and knowledge of complications (Pearson's $\chi^{2}(1)=1.3587$, Fisher's exact $p=0.343)$. However, women with type 1 diabetes demonstrated better knowledge of how pregnancy affects diabetes (26/61, $42.6 \%)$ than those with type 2 diabetics $(12 / 54,22.2 \%)$, (Pearson $\chi^{2}(1)=5.3881$, Fisher's exact $p=0.029)$. Women with type 1 diabetes reported a lower rate of contraception use than those not using insulin (type 2 diabetes) (Pearson $\chi^{2}(1)=3.3556$, Fisher's exact $\left.p=0.082\right)$.

Of the respondents, $90.4 \% \quad(n=104)$ had some knowledge of the complications of diabetes. It is noteworthy, however, that knowledge of pregnancy- and reproductive health-related complications was limited in our study population (Fig. 3). There was no association between the number of clinic visits and the knowledge participants displayed (Pearson $\chi^{2}(3)=0.7514$, Fisher's exact $p=0.881)$. Knowledge of complications of diabetes did not impact on whether patients were currently using contraception (Pearson $\chi^{2}(1)=0.4187$, Fisher's exact $\left.p=0.527\right)$.
Sixty-three respondents (54.8\%) were unable to identify how diabetes affected a pregnancy, and $77(67.0 \%)$ had no knowledge of how pregnancy affected diabetes. This was despite the fact that 102 participants $(88.7 \%)$ had access to diabetes clinic staff at their regular clinic visits, which occurred at least every 6 months. There was also no association between women's knowledge of how diabetes affects a pregnancy and current use of contraception (Pearson $\chi^{2}(1)=0.1488$, Fisher's exact $p=0.846$ ), and no association between knowledge of how pregnancy affects diabetes and current use of contraception (Pearson $\chi^{2}(1)=2.5499$, Fisher's exact $\left.p=0.150\right)$. We also showed no difference in contraception uptake among participants in relation to the number

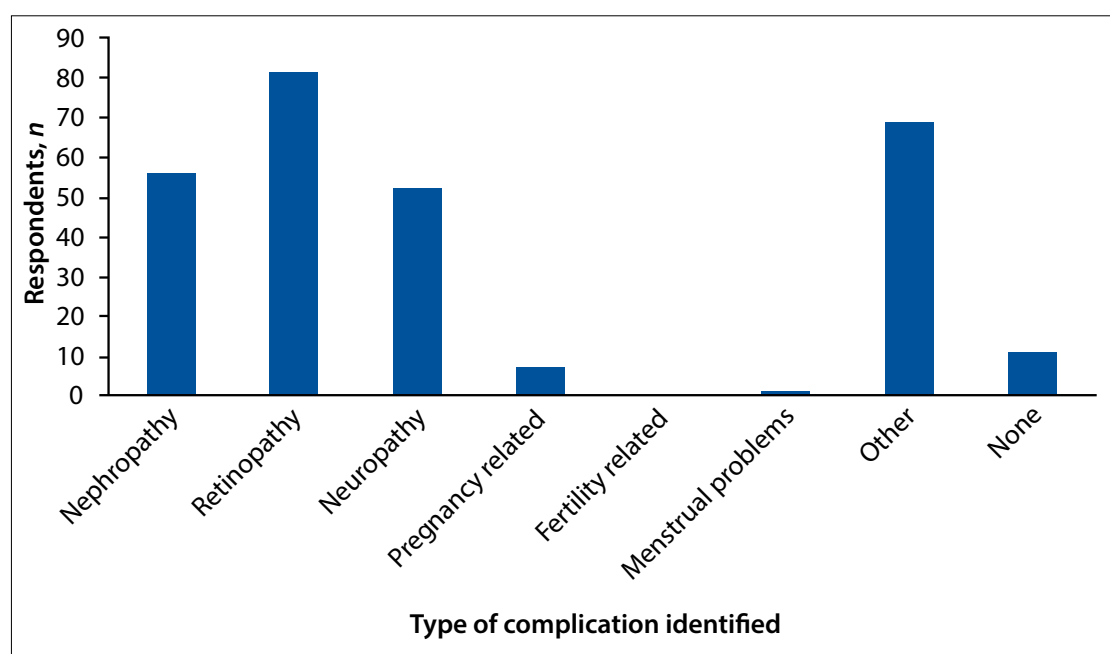

Fig. 3. Knowledge of complications associated with diabetes mellitus.

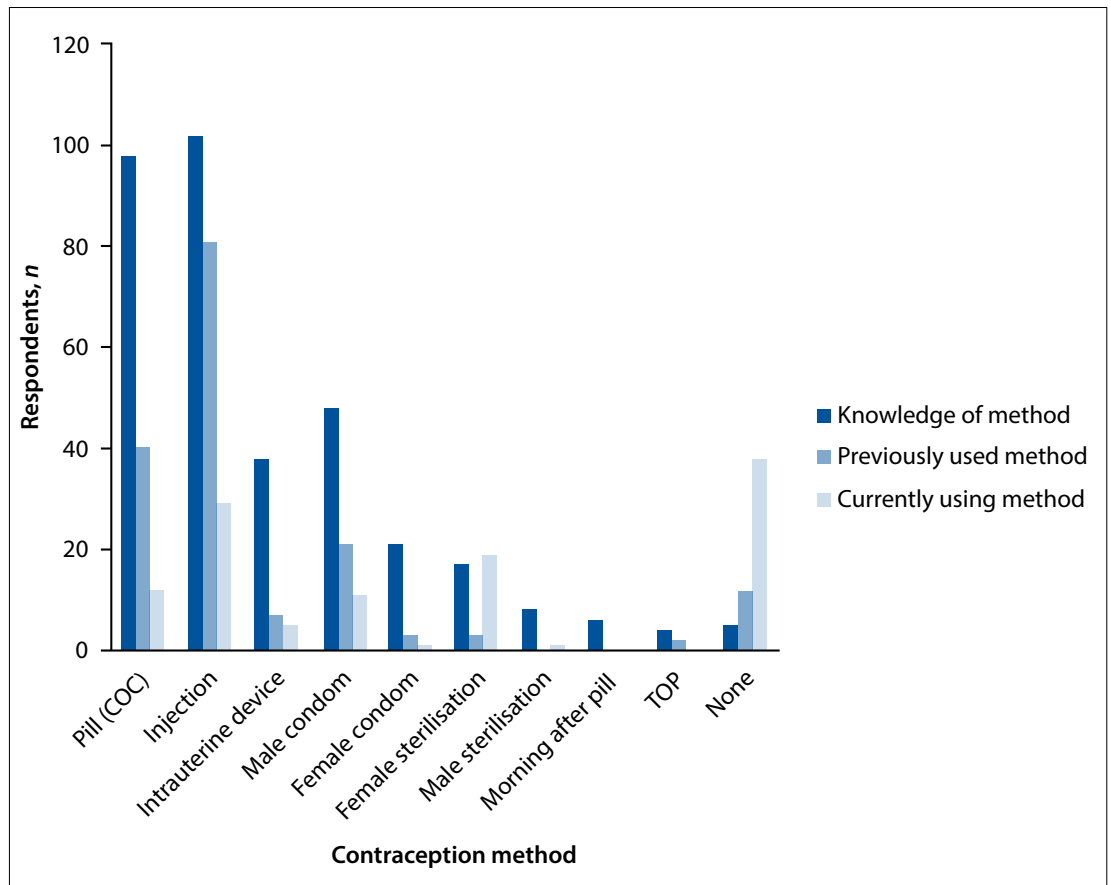

Fig. 4. Knowledge, previous use and current use of contraception by different methods. (COC = combined oral contraceptive; TOP = termination of pregnancy.) of clinic visits (Pearson $\chi^{2}(3)=3.66$, Fisher's exact $p=0.263$ ). with diabetes about their disease and their demographic profile, the only association we found was between marital status and knowledge of how diabetes affects a pregnancy, single women being less likely to know how diabetes affected a pregnancy (Pearson $\chi^{2}(2)=7.8510$, Fisher's exact $p=0.018$ ). Multiple confounders could be present if looking at marital status alone, and we attempted to adjust for age and parity.

\section{Contraceptive history}

Women were asked, without prompting, to name methods of contraception they had
In respect of knowledge of patients 
heard of, methods of contraception they had used in the past, and methods of contraception they were currently using (Fig. 4). Significant findings were that 12 participants (10.4\%) had never used contraception and a further $38(33.0 \%)$ were not currently using contraception.

Only $26.1 \%$ of the participants $(n=30)$ had previously received contraceptive advice at the diabetes clinic, and an overwhelming majority $(88.7 \%, n=102)$ felt uncomfortable about asking for advice there. Of particular note, $73.9 \%(n=85)$ indicated that they would like their diabetes doctor/sister to initiate a discussion about contraception. The most common place identified to access contraception was a family planning clinic $(54.8 \%, n=63)$, but other outlets were also mentioned. Significantly, no woman identified the diabetes clinics as a source of contraception.

We found no association between parity, level of education, emotional support or lifestyle and contraceptive use. In the case of marital status, we found an increased association between being single and not using contraception when compared with married women or women in stable relationships (Pearson $\chi^{2}(2)=7.9214$, Fisher's exact $p=0.025$ ).

\section{Discussion}

The themes that emerged from this study were that women attending outpatient diabetes clinics in our services have a limited knowledge of the impact of diabetes on pregnancy outcome, as well as on the impact of pregnancy on disease progression. Counselling on reproductive health, contraception and the effect of diabetes in pregnancy appears to be inadequate. This is supported by the fact that a large proportion of participants in our study (34/77, 44.2\%) reported unintended pregnancies, indicating that their use of contraception was suboptimal. Although women with type 1 diabetes had a better understanding than those with type 2 diabetes of how pregnancy affects diabetes, there was still low use of contraception among these patients that we cannot explain.

Studies in the USA have reported that in general women at highest risk of unintended pregnancies are unmarried and of low socioeconomic status, have a low literacy level, and belong to minority groups. These women also tend to have poor glycaemic control and are less likely than other women to talk to their doctor before planning a pregnancy. They usually present for antenatal care at a late gestational age, which makes it difficult to provide optimal care. ${ }^{[8-10]}$

Our study showed an association between younger age and unintended pregnancies, as well as between single status and unintended pregnancies. It is a misconception that women who are not currently in a sexual relationship do not need contraceptive advice. Sexual activity is a status that can change suddenly, and all women of reproductive age, particularly those with high-risk medical conditions, should have contraceptive counselling irrespective of their current status as defined by the SADHS. Waiting till they become sexually active can be too late for some.

Women with diabetes need to remain on contraception until optimal glucose control is achieved, chronic medications are adjusted and lifestyle adjustments (e.g. smoking cessation) have been made; this should be reiterated at every visit.

Adequate glycaemic control at the time of conception and during the period of organogenesis in early pregnancy reduces the risks of congenital malformations and spontaneous miscarriages to virtually the same as those in women without diabetes. International recommendations are haemoglobin Alc levels $<7 \%$ (American Diabetes Association (ADA) guidelines) and $<6.1 \%$ (UK National Institute of Clinical Excellence (NICE) guidelines). ${ }^{[1,12]}$
All but one of the women in our study were using some form of medical treatment (114/115). These included oral agents, insulin, antihypertensives and statins, some of which need to be revised during pregnancy. Despite the risk associated with pregnancy, our data showed that 12 of the participants $(10.4 \%)$ had never used contraception, and a further 38 (33.0\%) were not currently using contraception. The importance of contraception did not appear to feature in the otherwise excellent counselling they received in the diabetes clinics.

We found that only 30 of the study participants $(26.1 \%)$ had previously received any contraceptive or pregnancy planning advice at the diabetes clinics. Varughese et al. ${ }^{[13]}$ concluded in a 2007 review of women attending adult general diabetes clinics in Telford, UK, that the aim of diabetes services should be to enable the pregnant woman with diabetes to present to her obstetrician with such well-controlled levels that her pregnancy will proceed without any complications.

Studies by Schwarz et al..$^{[9]}$ and Chuang et al. ${ }^{[14]}$ both demonstrated that despite relatively frequent contact with healthcare providers, adolescent women with diabetes rarely identified a doctor or nurse as a major source of information about contraception and furthermore that they did not feel comfortable asking a healthcare professional for advice about contraception. Our findings were similar in that despite the fact that $89 \%$ of the participants were attending specialist diabetes clinics two or more times a year for routine visits, none identified the clinic as a point of contact for their contraception needs.

It is of concern that women attending diabetes clinics three or four times a year may neglect their family planning clinic appointments, as they perceive these to be less important. It falls to the doctor or nurse in the general clinics to make access to these services as easy as possible, especially when they can be accessed in the same facility.

Women with chronic medical conditions are at an increased risk of pregnancy-related complications, yet little research (with the exception of research among HIV-positive patients) has addressed how they perceive their pregnancy-associated risks or make reproductive health choices.

Crede et al. ${ }^{[15]}$ showed in a study among postpartum women in Cape Town that the majority of the subjects in both the HIV-positive and HIV-negative sample groups indicated that their last pregnancy had been unintended (61.6\% and $63.2 \%$, respectively). Nearly $90 \%$ of women in both groups reported they were using a modern method of contraception $(89.8 \%$ of HIV-positive and $89.0 \%$ of HIV-negative women), but typically this was a short-acting method with a higher failure rate than the long-acting reversible contraceptives. This was very similar to the types of contraception identified by the participants in our study.

Women with diabetes are generally aware that they have a risk of complications, but they have very little knowledge about the risk of pregnancy-related complications. ${ }^{[14]}$ This was illustrated in our study, where 104 of the 115 patients interviewed had some knowledge of the complications of diabetes. When pregnancy- and reproductive health-related complications were looked at more specifically, however, knowledge was limited: 63 respondents (54.8\%) were unable to identify how diabetes affected a pregnancy, and $77(67.0 \%)$ were unable to suggest any way in which pregnancy could affect diabetes.

\section{Conclusion}

Contraceptive advice and pregnancy planning discussions should form part of the clinical review of every woman in the general diabetes clinic, and should be initiated in their early reproductive years. ${ }^{[13]}$

Ideally, women with diabetes should conceive when they are best prepared for a pregnancy, financially, socially and medically. 
Improved patient motivation, intensive input from diabetes services, and interdisciplinary interactions between the obstetric and medical teams managing these high-risk patients should form the essential components of care if we are to successfully address the challenge of reducing the current unacceptably high rates of maternal and perinatal morbidity and mortality in SA.

Acknowledgements. We appreciate the support we received from the staff in the diabetes clinics and thank the Department of Internal Medicine, GSH, for allowing us to conduct this research within their clinics. This research was partly sponsored by the JS Scratchley Trust.

\section{References}

1. International Diabetes Federation. IDF Diabetes Atlas. 6th ed. Brussels, Belgium: IDF, 2013. http:// www.idf.org/diabetesatlas (accessed 17 February 2015).

2. Federation of European Nurses in Diabetes and International Diabetes Federation - Europe. Diabetes: The Policy Puzzle: Is Europe making progress? 2nd ed. International Diabetes Federation, 2008:3-6. https://www.idf.org/sites/default/files/EU-diabetes-policy-audit-2008\%20-2nd\%20edition.pdf (accessed 11 August 2015).

3. Kitzmiller JL, Block JM, Brown FM, et al. Managing pre-existing diabetes for pregnancy: Summary of evidence and consensus recommendations for care. Diabetes Care 2008;31(5):1060-1079. [http:// .

Walkinshaw SA. Type 1 and type 2 diabetes and pregnancy. Curr Obstet Gynaecol 2004;14(6):375-386. [http://dx.doi.org/10.1016/j.curobgyn.2004.07.002]
5. National Committee on Confidential Enquiries into Maternal Deaths. Saving Mothers 2005-2007: Fourth Report on Confidential Enquiries into Maternal Deaths in South Africa: Expanded Executive Summary. Pretoria: Department of Health, 2009.

6. McDonagh M. Is antenatal care effective in reducing maternal morbidity and mortality? Health Policy Plan 1996;11(1):1-15. [http://dx.doi.org/10.1093/heapol/11.1.1]

7. Department of Health, Medical Research Council, OrcMacro. South Africa Demographic and Healt Survey 2003. Pretoria: Department of Health, 2007.

8. Dunlop AL, Jack BW, Bottalico JN, et al. The clinical content of preconception care: Women wit chronic medical conditions. Am J Obstet Gynecol Supplement to December 2008;199(6,Suppl B):s310-s327. [http://dx.doi.org/10.1016/j.ajog.2008.08.031]

9. Schwarz EB, Sobota M, Charron-Prochownik D. Perceived access to contraception among adolescents with diabetes: Barriers to preventing pregnancy complications. Diabetes Educ 2010;36(3):489. [http:// dx.doi.org/10.1177/0145721710365171] [PMID: 20332282]

10. Henshaw SK. Unintended pregnancy in the United States. Fam Plann Perspect 1998;30(1):24-29,46. [http://dx.doi.org/10.2307/2991522]

11. American Diabetes Association. Standards of medical care in diabetes - 2010. Diabetes Care 2010;33(1):s11-s61. [http://dx.doi.org/10.2337/dc10-S011]

Int of diabetes from preconception to the (o) the postnatal period: Summary of NICE guidance. BM] 2008;336(7646):714-717. [http://dx.do org/10.1136/bmj.39505.641273.AD]

3. Varughese GI, Chowdhury SR, Warner DP, Barton DM. Preconception care of women attending adul general diabetes clinics - are we doing enough? Diabetes Res Clin Pract 2007;76(1):142-145. [http:// dx.doi.org/10.1016/j.diabres.2006.07.025

14. Chuang CH, Velott DL, Weisman CS. Exploring knowledge and attitudes related to pregnancy and preconception health in women with chronic medical conditions. Matern Child Health 2010;14(5):713-719. [http://dx.doi.org/10.1007/s10995-009-0518-6]

15. Crede S, Hoke T, Contant D, et al. Factors impacting knowledge and use of long acting and permanent contraceptive methods by postpartum HIV positive and negative women in Cape Town, South Africa: A cross-sectional study. BMC Public Health 2012;12:197. [http://dx.doi.org/10.1186/1471-2458-12-197]

Accepted 30 June 2015 